\title{
On even-dimensional fibred knots obtained by plumbing
}

\author{
By DANIEL LINES \\ Institut de mathématiques, Université de Neuchâtel, Switzerland
}

(Received 15 October 1985)

\begin{abstract}
Introduction
In this article we extend the study of 'plumbing' initiated in [10] to the case of fibred even-dimensional knots. Plumbing is a geometric operation on the fibre-surfaces of two fibred knots of the same dimension that produces another such knot.

All knots obtained in this way are 'free' (i.e. simple in a strong sense) (cf. § 1) and non-spherical knots occur. We classify these fibred free even-dimensional knots algebraically by means of equivalence classes of triples of matrices (cf. §2). This classification is due to Kojima [7] in the case of spherical knots but some of our proofs differ from [7].

As a result of the classification, we show that there are exactly 4 types of nontrivial knots of smallest possible rank.

In $\$ 3$ we define the concept of plumbing for even-dimensional knots, characterize the triples of matrices associated with knots obtained by plumbing (Theorem 3.4) and show that there exist infinitely many free fibred knots that are NOT obtained by this process (Theorem 3.6).

Spinning an odd-dimensional simple fibred knot $K$ produces an even-dimensional free fibred knot $\sigma(K)$ (cf. §4). We compute the matrix triple associated to such a spun knot and recover in this context a result of $C$. Kearton [4]. In particular we show that if $K$ is obtained by plumbing, so is the spun knot $\sigma(K)$.
\end{abstract}

\section{Definitions}

All manifolds considered are differentiable; $q$ will always denote an integer $\geqslant 4$. A (2q)-dimensional knot is a closed oriented submanifold $K$ of dimension $2 q$ of the oriented $(2 q+2)$-dimensional sphere $S^{2 q+2}$ such that $K$ is $(q-2)$-connected.

The knot $K$ is called spherical if $K$ is homeomorphic to $S^{2 q}$.

$K$ is a free knot if $\pi_{i}\left(S^{2 q+2} \backslash K\right) \cong \pi_{i}\left(S^{1}\right)$ for $i \leqslant q-1$ and $\pi_{q}\left(S^{2 q+2} \backslash K\right)$ is a torsion-free abelian group.

It is well known that every $(2 q)$-dimensional knot $K$ bounds a Seifert surface $V$, i.e. a $(2 q+1)$-dimensional oriented submanifold of $S^{2 q+2}$. The normal bundle to $V$ in $S^{2 q+2}$ is trivial and there are two maps $i_{+}$(resp. $\left.i_{-}\right): V \rightarrow S^{2 q+2} \backslash V$ obtained by pushing points of $V$ in the positive (resp. negative) direction of this bundle.

A free Seifert surface $V$ for $K$ is a $(q-1)$-connected Seifert surface such that $\pi_{q}(V) \simeq H_{q}(V)$ is a free abelian group.

LeMMA 1·1. $K$ is a free knot if and only if $K$ admits a free Seifert surface. 
Proof. Let $K$ be a free knot and $V$ be a Seifert surface for $K$. By ([8], theorem 2) we may suppose that $V$ is $(q-1)$-connected; ([8], lemma 3) implies that

$$
i_{ \pm}: H_{q}(V) \rightarrow H_{q}\left(S^{2 q+2} \backslash V\right)
$$

are injective. ([5], p. 246) shows that under these circumstances the torsion subgroups of $\pi_{q}\left(S^{2 q+2} \mid K\right)$ and $H_{q}\left(S^{2 q+2} \mid V\right)$ are isomorphic, hence $V$ is $(q-1)$-connected and $H_{q}(V)$ is free abelian.

Conversely, suppose that $K$ admits a free Seifert surface $V$. Let $\mathcal{X}$ be the infinite cyclic cover of $K$ and set $Y=S^{2 q+2} \backslash V$. The standard construction of $X$ from copies of $Y$ implies that $\pi_{i}\left(S^{2 q+2} \backslash K\right)$ is isomorphic to $\pi_{i}\left(S^{1}\right)$ for $i \leqslant q-1$ (cf. [6], $\left.\S 2\right)$. By Alexander duality and universal coefficients $H_{q}(Y)$ is a free abelian group; the kernels of $i_{ \pm}: H_{q}(V) \rightarrow H_{q}(Y)$ are therefore generated by indivisible elements. Applying ([8], lemma 3) to these elements, one gets a new free Seifert surface $V^{\prime}$ for $K$ such that $i_{ \pm}: H_{q}\left(V^{\prime}\right) \rightarrow H_{q}\left(S^{2 q+2} \backslash V^{\prime}\right)$ are both injective. The argument of ([5], p. 246) shows again that $\pi_{q}\left(S^{2 q+2} \mid K\right)$ is torsion-free.

Let $T_{K}$ be the tubular neighbourhood of $K ; T_{K}$ is a trivial disc bundle over $K$.

Definition. $K$ is fibred if there is a trivialization $\Phi: T_{K} \rightarrow K \times D^{2}$ such that $p r_{2} \circ \Phi: \partial T_{K} \rightarrow S^{1}$ extends to a locally trivial fibration of $S^{2 q+2} \mid T_{K}$ over $S^{1}$. The inverse image of a point is (after collaring) a Seifert surface for $K$ called the fibre-surface.

Note that if $K$ is a free fibred knot and $F$ is the fibre-surface, $F$ is necessarily a free Seifert surface for $K$ since $F$ has the homotopy type of the infinite cyclic cover $\widetilde{X}$ of $K$.

Using Lefschetz duality and universal coefficients one easily proves the following:

LEMMA 1.2. If $K$ is a (2q)-dimensional knot bounding a free Seifert surface $V$, then:

(i) $\tilde{H}_{k}(K)=0$ for $0 \leqslant k \leqslant q-2$ and $q+2 \leqslant k<2 q$,

(ii) $\tilde{H}_{k}(V)=\hat{H}^{k}(V)=0$ for $k \leqslant q-1$ and $k \geqslant q+2$.

$H_{k}(V)$ and $H^{k}(V)$ are free abelian for $k=q, q+1$.

Remark. By a theorem of Smale [12], $V$ is obtained from the disc $D^{2 q+1}$ by attaching handles of index $q$ and $q+1$.

The following lemma is proved using Alexander duality.

Lemma 1.3. Let $V$ be a free Seifert surface in $S^{2 q+2}$ and $W$ be a tubular neighbourhood of $V$. Set $W^{\prime}=S^{2 q+2} \mid W^{W}$. The two linking forms

$$
\begin{aligned}
L: H_{q}(W) \times H_{q+1}\left(W^{\prime}\right) & \rightarrow \mathbb{Z}, \\
L^{\prime}: H_{q}\left(W^{\prime}\right) \times H_{q+1}(W) & \rightarrow \mathbb{Z},
\end{aligned}
$$

are unimodular, in particular $H_{q}\left(W^{\prime}\right)$ and $H_{q+1}\left(W^{\prime}\right)$ are free abelian groups.

The following bilinear forms are attached to a free Seifert surface $V$ : the intersection form

$$
I: H_{q}(V) \times H_{q+1}(V) \rightarrow \mathbb{Z}
$$

the (homological) Seifert forms

$$
\begin{array}{ll}
A_{+}: H_{q}(V) \times H_{q+1}(V) \rightarrow \mathbb{Z} & (x ; y) \rightarrow L\left(x ; i_{+} y\right), \\
A_{-}: H_{q}(V) \times H_{q+1}(V) \rightarrow \mathbb{Z} & (x ; y) \rightarrow L\left(x ; i_{-} y\right) .
\end{array}
$$

Note that $I(x ; y)=L\left(x ; i_{+} y-i_{-} y\right)=A_{+}(x ; y)-A_{-}(x ; y)$. 
LEMMA 1.4. Let $K$ be a (2q)-dimensional knot bounding a free Seifert surface $V$ with intersection form $I$; then $K$ is spherical if and only if $I$ is unimodular.

Proof. Consider the two relative intersection forms of $V$

$$
\begin{aligned}
J: H_{q}(V) \times H_{q+1}(V ; \partial V) & \rightarrow \mathbb{Z}, \\
J^{\prime}: H_{q}(V ; \partial V) \times H_{q+1}(V) & \rightarrow \mathbb{Z} .
\end{aligned}
$$

Since $H_{q}(V)$ and $H_{q}(V ; \partial V)$ are free abelian, Lefschetz duality implies that $J$ and $J^{\prime}$ are unimodular. Furthermore $I(x ; y)=J(x ; p y)=J^{\prime}\left(p^{\prime} x ; y\right)$ where

$$
p: H_{q+1}(V) \rightarrow H_{q+1}(V ; \partial V) \text { and } p^{\prime}: H_{q}(V) \rightarrow H_{q}(V ; \partial V)
$$

are the natural maps. This shows that $I$ is unimodular if and only if $p$ and $p^{\prime}$ are isomorphisms. The long exact sequence of the pair $(V ; \partial V)$ shows that the latter is equivalent to $H_{k}(K)=0$ for $q-1 \leqslant k \leqslant q+1$. Since $q \geqslant 3$, this condition is satisfied if and only if $K$ is homeomorphic to $S^{2 q}$.

LEMMa 1-5. Let $V$ be a free Seifert surface in $S^{2 a+2}$. Then $i_{ \pm}: H_{k}(V) \rightarrow H_{k}\left(S^{2 q+2} \backslash V\right)$ are isomorphisms for $k=q, q+1$ if and only if $A_{+}$and $A_{-}$are unimodular.

Proof. $i_{ \pm}: H_{q+1}(V) \rightarrow H_{q+1}\left(S^{2 q+2} \backslash V\right)$ are isomorphisms if and only if

$$
A_{ \pm}(x ; y)=L\left(x ; i_{ \pm} y\right)
$$

are unimodular forms, by Lemma 1.3. Conversely if $A_{+}$and $A_{-}$are unimodular, to show that $i_{ \pm}: H_{q}(V) \rightarrow H_{q}\left(S^{2 q+2} \backslash V\right)$ are also isomorphisms, consider the equality

$$
L^{\prime}\left(i_{ \pm} x ; y\right)=L\left(x ; i_{\mp} y\right)=A_{\mp}(x ; y)
$$

and recall that $L^{\prime}$ is unimodular.

Proposition 1.6. Let $K$ be a (2q)-dimensional knot bounding a free Seifert surface $V$ and let $A_{+}$and $A_{-}$be the associated Seifert forms. Then $K$ is fibred with fibre-surface $V$ if and only if $A_{+}$and $A_{-}$are unimodular.

Proof. If $K$ is fibred with fibre-surface $V, i_{+}$and $i_{-}$are homotopy equivalences and induce isomorphisms in homology. Lemma 1.5 shows that $A_{+}$and $A_{-}$are unimodular. Conversely if $A_{+}$and $\dot{A}_{-}$are unimodular $i_{ \pm}: H_{k}(V) \rightarrow H_{k}\left(S^{2 a+2} \backslash V\right)$ are isomorphisms for all $k$. Denote by $T_{V}$ a tubular neighbourhood of $V$ in $S^{2 q+2}$. Since $V$ and $S^{2 q+2} \backslash \dot{T}_{V}$ are simply connected, the $h$-cobordism theorem implies that $S^{2 q+2} \backslash \dot{T}_{V}$ is diffeomorphic to $V \times[0,1]$. This shows that $K$ is fibred with fibre-surface $V$.

We now recall the definition and some properties of the homotopy linking number.

Let $f: S^{q+1} \hookrightarrow S^{2 q+2}$ and $g: S^{q+1} \hookrightarrow S^{2 q+1}$ be two embeddings with disjoint image. Since $g\left(S^{q+1}\right)$ is unknotted in $S^{2 q+2}, \pi_{q+1}\left(S^{2 q+2} \backslash g\left(S^{q+1}\right)\right)$ is isomorphic to $\pi_{q+1}\left(S^{q}\right)$ which is cyclic of order 2. Consider $[f] \in \pi_{q+1}\left(S^{2 q+2} \backslash g\left(S^{q+1}\right)\right)$ and define the homotopy linking number $\mathscr{L}(f ; g)$ to be 0 if $f$ represents the trivial element, 1 otherwise. It can be shown that $\mathscr{L}$ is symmetric in $f$ and $g$.

LeMma 1·7. (i) Let f,g: $S^{q+1} \hookrightarrow S^{2 q+2}$ be embeddings with disjoint image, let

$$
\lambda: D^{a+2} \hookrightarrow S^{2 q+2}
$$

be an embedding such that $\lambda \mid \partial D^{q+2}=g$ and such that $\lambda$ is in general position with $f$. Let $\Delta$ be $a(2 q+2)$-ball in $S^{2 q+2}$ engulfing the intersections of $\lambda$ and $f$ such that

$$
\Delta(\lambda)=\lambda\left(D^{a+2}\right) \cap \Delta \text { is a properly embedded }(q+2)-d i s c,
$$


and $\Delta(f)=f\left(S^{q+1}\right) \cap \Delta$ is a properly embedded $(q+1)$-disc.

Consider $[\partial \Delta(f)]$ as an element of $\pi_{q}(\partial \Delta \mid \partial \Delta(\lambda)) \simeq \mathbb{Z} / 2 ;$ then $\mathscr{L}(f ; g)$ and $[\partial \Delta(f)]$ represent the same element in $\mathbb{Z} / 2$.

(ii) Let $F, G$ : $D^{a+2} \hookrightarrow D^{2 q+3}$ be proper embeddings such that $F\left|\partial D^{a+2}=f, G\right| \partial D^{a+2}=g$ and suppose that $F\left(D^{q+2}\right) \cap G\left(D^{q+2}\right)=\varnothing$; then $\mathscr{L}(f ; g)=0$.

Proof. (i) Since $q \geqslant 3$, the sphere pairs $\left(S^{2 q+2} ; g\left(S^{q+1}\right)\right)$ and $\left(\partial \Delta^{2 q+2} ; \partial \Delta(\lambda)\right)$ are unknotted, so that $S^{q q+2} \mid g\left(S^{q+1}\right)$ (resp. $\left.\partial \Delta \mid \partial \Delta(\lambda)\right)$ has the homotopy type of $S^{q+1}$ (resp. $\left.S^{q}\right)$. There is a suspension isomorphism $\pi_{q}(\partial \Delta \mid \partial \Delta(\lambda)) \rightarrow \pi_{q+1}\left(S^{2 q+2} \mid g\left(S^{q+1}\right)\right)$ which sends the homotopy class of $\partial \Delta(f)$ to the homotopy class of $f$. This shows that $\mathscr{L}(f ; g)$ and $\partial \Delta(f)$ correspond to the same element of $\mathbb{Z} / 2$.

(ii) The inclusion of $S^{2 q+2} \backslash g\left(\mathcal{S}^{q+1}\right)$ in $D^{2 q+3} \backslash G\left(D^{q+2}\right)$ gives an exact sequence:

$$
\pi_{q+2}\left(D^{2 q+3} \backslash G\left(D^{q+2}\right) ; S^{2 q+2} \mid g\left(S^{q+1}\right)\right) \stackrel{\partial}{\rightarrow} \pi_{q+1}\left(S^{2 q+2} \mid g\left(S^{q+1}\right)\right) \stackrel{i *}{\rightarrow} \pi_{q+1}\left(D^{2 q+3} \backslash G\left(D^{q+2}\right)\right) .
$$

Since $q \geqslant 3$, the ball pair $\left(D^{2 q+3} ; G\left(D^{q+2}\right)\right)$ is unknotted, $i_{*}$ is an isomorphism and $\partial$ is the zero homomorphism. $F$ represents an element in $\pi_{q+2}\left(D^{2 q+3} \backslash G\left(D^{q+2}\right) ; \$^{2 q+2} \backslash g\left(S^{q+1}\right)\right)$ and clearly $\partial[F]=[f]$. This shows that $\mathscr{L}(f ; g)=0$.

Let $K$ be a $2 q$-dimensional knot bounding a free Seifert surface $V$ and let $x$ and $y$ be elements of $\pi_{q+1}(V)$. Since $q \geqslant 4$, we can represent $x$ and $y$ by embeddings

$$
\phi_{x}, \phi_{y}: S^{q+1} \hookrightarrow V
$$

(cf. [2]). Set $\mathscr{A}(x ; y)=\mathscr{L}\left(\phi_{x} ; i_{+} \circ \phi_{y}\right)$. This defines a bilinear map

$$
\mathscr{A}: \pi_{q+1}(V) \times \pi_{q+1}(V) \rightarrow \mathbb{Z} / \mathbf{2}
$$

and induces a bilinear form over $\mathbb{F}_{2}$ on the $\mathbb{F}_{2}$-vector space $\pi_{q+1}(V) \otimes \mathbb{F}_{2}$, which we still denote by $\mathscr{A}$ and call the homotopy Seifert form of $V$.

A theorem of Whitehead ([14], chapter XII, theorem 3.12) shows that the following sequence is exact:

$$
0 \rightarrow H_{q}(V) / 2 H_{q}(V) \stackrel{\eta}{\rightarrow} \pi_{q+1}(V) \stackrel{h}{\rightarrow} H_{q+1}(V) \rightarrow 0,
$$

where $h$ is the Hurewicz map and $\eta$ is defined as follows: let $\eta_{0}: S^{q+1} \rightarrow S^{q}$ represent the non-trivial element of $\pi_{q+1}\left(S^{q}\right)$, and let $f: S^{q} \rightarrow V$ represent any element $\alpha$ of $H_{q}(V) / 2 H_{q}(V)$. Set $\eta(\alpha)$ to be the homotopy class of $f \circ \eta_{0}$.

Let

$$
\begin{gathered}
\left\{\alpha_{k}\right\} k=1, \ldots, r \quad \text { be a } \mathbb{Z} \text {-basis of } H_{q}(V), \\
\left\{\beta_{l}\right\} l=1, \ldots, s \quad \text { be a } \mathbb{Z} \text {-basis of } H_{q+1}(V)
\end{gathered}
$$

and $\beta_{l}^{\#}$ be any lift of $\beta_{l}$ in $\pi_{q+1}(V)$. Set $\alpha_{k}^{\#}=\eta\left(\alpha_{k}\right) \cdot \pi_{q+1}(V) \otimes \mathbb{F}_{2}$ is a $\mathbb{F}_{2}$-vector space of dimension $r+s$ with basis $\left\{\alpha_{k}^{\#} ; \beta_{l}^{\#}\right\}$. Define an $s \times s$ matrix $B$ with coefficients in $\mathbb{F}_{2}$ by the formula $B_{i j}=\mathscr{A}\left(\beta_{i}^{\#} ; \beta_{j}^{\#}\right)$. To simplify the notation we denote by the same symbol a bilinear form and its matrix with respect to a given basis.

LeMmA 1.8. The matrix of the $\mathbb{F}_{2}$-bilinear form $\mathscr{A}$ with respect to the basis above is

$$
\left(\begin{array}{cc}
0 & A_{+} \\
A_{-}^{T} & B
\end{array}\right)
$$

Proof. Suppose that $x$ and $y$ in $\pi_{q+1}(V)$ are represented by $\phi_{x}$ and $\phi_{y}: S^{q+1} \rightarrow V$ where $\phi_{x}=f_{x} \circ \eta_{0}$ and $f_{x}: S^{q} \hookrightarrow V, \phi_{y}: S^{q+1} \hookrightarrow V$ are embeddings. The Whitehead exact 
sequence applied to the space $S^{2 q+2} \backslash i_{+} \phi_{y}\left(S^{q+1}\right)$ shows that $\eta$ sends the homology class carried by $f_{x}\left(S^{q}\right)$ to the homotopy class of $\phi_{x}$, therefore

$$
\mathscr{A}(x ; y)=L\left(f_{x}\left(S^{q}\right) ; i_{+} \phi_{y}\left(S^{q+1}\right)\right) \quad(\bmod 2)
$$

where $L$ denotes the ordinary linking number in $S^{2 q+2}$. It is then easy to see that $\mathscr{A}\left(\alpha_{i}^{\#} ; \alpha_{j}^{\#}\right)=0$ and $\mathscr{A}\left(\alpha_{i}^{\#} ; \beta_{j}^{\#}\right)=A_{+}\left(\alpha_{i} ; \beta_{j}\right)(\bmod 2)$. Pushing the representatives of the homotopy classes in the negative direction of the tubular neighbourhood of $V$ and applying the same argument shows that $\mathscr{A}\left(\beta_{i}^{\#} ; \alpha_{j}^{\#}\right)=A_{-}\left(\alpha_{j} ; \beta_{i}\right)(\bmod 2)$.

Recall that C.T.C. Wall ([13], p. 256) has defined a generalized intersection number $\mathscr{I}: \pi_{q+1}(V) \times \pi_{q+1}(V) \rightarrow \pi_{q+1}\left(S^{q}\right) \simeq \mathbb{Z} / 2$. In exactly the same way one can define a relative bilinear map $\mathscr{J}: \pi_{q+1}(V) \times \pi_{q+1}(V ; \partial V) \rightarrow \pi_{q+1}\left(S^{q}\right)$. These two maps induce bilinear forms over $\mathbb{F}_{2}$ :

$$
\begin{aligned}
& \mathscr{I}: \pi_{q+1}(V) \otimes \mathbb{F}_{2} \times \pi_{q+1}(V) \otimes \mathbb{F}_{2} \rightarrow \mathbb{F}_{2}, \\
& \mathscr{J}: \pi_{q+1}(V) \otimes \mathbb{F}_{2} \times \pi_{q+1}(V ; \partial V) \otimes \mathbb{F}_{2} \rightarrow \mathbb{F}_{2} .
\end{aligned}
$$

Remark 1.9. $\mathscr{I}$ and $\mathscr{J}$ can be computed as follows (cf. [7], lemma 1). Represent $x$ and $y$ in $\pi_{q+1}(V)$ by embeddings $f, g: S^{q+1} \hookrightarrow V$ in general position and engulf their intersection in the interior of a $(2 q+1)$ - ball $\Delta$ contained in $V$ such that $\Delta(f)=f\left(S^{q+1}\right) \cap \Delta$ and $\Delta(g)=g\left(S^{q+1}\right) \cap \Delta$ are properly embedded discs; $\partial \Delta(f)$ is an element of

$$
\pi_{Q}(\partial \Delta \mid \partial \Delta(g)) \simeq \mathbb{Z} / 2
$$

Then $\mathscr{I}(x ; y)$ and $\partial \Delta(f)$ correspond to the same element in $\mathbb{Z} / 2$. The procedure for $\mathscr{J}$ is the same except that $y$ is represented by a properly embedded dise in $V$.

Lemma 1 10. The following properties hold:

(i) $\mathscr{I}(x ; y)=\mathscr{I}(x ; p y)$ where $p: \pi_{q+1}(V) \rightarrow \pi_{q+1}(V ; \partial V)$ is the natural map.

(ii) $\mathscr{I}=\mathscr{A}-\mathscr{A}^{T}$.

(iii) $\mathscr{J}$ is non singular.

Proof. (i) Is evident from Remark 1.9.

(ii) Let $x$ and $y$ be elements of $\pi_{q}(V)$; we have

$$
\left(\mathscr{A}-\mathscr{A}^{T}\right)(x ; y)=\mathscr{L}\left(x ; i_{+} y\right)-\mathscr{L}\left(y ; i_{+} x\right)=\mathscr{L}\left(x ; i_{+} y-i_{-} y\right) .
$$

Let $f_{x}, f_{y}: S^{q+1} \hookrightarrow V$ represent $x$ and $y$ and let $\Delta$ be $a(2 q+1)$-ball in $V$ engulfing all intersections of $f_{x}$ and $f_{y}$ such that $\Delta\left(f_{x}\right)=f_{x}\left(S^{q+1}\right) \cap \Delta$ and $\Delta\left(f_{y}\right)=f_{y}\left(S^{q+1}\right) \cap \Delta$ are properly embedded $(q+1)$-discs. Thicken $\Delta$ to a $(2 q+2)$-ball $\Delta^{\prime}$ along the normal bundle to $V$ and denote by $C$ the cylinder spanning $i_{+} f_{y}\left(S^{q+1}\right)$ and $i_{-} f_{y}\left(S^{q+1}\right)$ in the tubular neighbourhood of $V$. By deleting a neighbourhood of an arc joining $i_{+} f_{y}\left(S^{q+1}\right)$ to $i_{-} f_{y}\left(S^{q+1}\right)$ and missing $f_{x}\left(S^{q+1}\right)$ one produces a $(q+2)$-dise $D$ such that

$$
f_{x}\left(S^{q+1}\right) \cap D=f_{x}\left(S^{q+1}\right) \cap f_{y}\left(S^{q+1}\right) .
$$

Lemma 1.7 shows that $\mathscr{L}\left(x ; i_{+} y-i_{-} y\right)$ is represented by $\Delta\left(f_{x}\right)$ in $\pi_{q}\left(\partial \Delta^{\prime} \backslash \partial \Delta^{\prime} \cap D\right)$ and Remark 1.9 that $\mathscr{I}(x ; y)$ is represented by $\Delta\left(f_{x}\right)$ in $\pi_{q}(\partial \Delta \backslash \partial \Delta \cap D)$. Since the two pairs $(\partial \Delta ; \partial \Delta \cap D)$ and $\left(\partial \Delta^{\prime} ; \partial \Delta^{\prime} \cap D\right)$ are unknotted the inclusion of $\partial \Delta \mid \partial \Delta \cap D$ in $\partial \Delta^{\prime} \mid \partial \Delta^{\prime} \cap D$ induces an isomorphism between the homotopy groups. This proves that

$$
\mathscr{I}(x ; y)=\mathscr{L}\left(x ; i_{+} y-i_{-} y\right)
$$


(iii) Using ([14], chapter VII, theorem 7.12) one sees that $\pi_{k}(V ; \partial V)$ is isomorphic to $\pi_{k}(V / \partial V)$ for $k \leqslant q+1$. Therefore there is a relative Whitehead exact sequence

$$
0 \rightarrow H_{q}(V ; \partial V) / 2 H_{q}(V ; \partial V) \rightarrow \pi_{q+1}(V ; \partial V) \rightarrow H_{q+1}(V ; \partial V) \rightarrow 0 .
$$

The following diagram of $\mathbb{F}_{2}$ vector spaces clearly commutes:

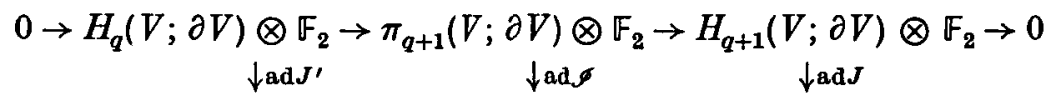

$$
\begin{aligned}
& 0 \leftarrow \operatorname{Hom}\left(H_{q+1}(V) ; \mathbb{F}_{2}\right) \leftarrow \operatorname{Hom}\left(\pi_{q+1}(V) ; \mathbb{F}_{2}\right) \leftarrow \operatorname{Hom}\left(H_{q}(V) ; \mathbb{F}_{2}\right) \leftarrow 0
\end{aligned}
$$

Since ad $J$ and ad $J^{\prime}$ are isomorphisms, so is ad $\mathscr{J}$.

\section{Classification of free fibred knots}

Let $K$ be a $2 q$-dimensional free fibred knot with fibre-surface $F$ and infinite cyclic cover $\bar{X} . F$ and $\tilde{X}$ have the same homotopy type and $H_{q}(F)$ is isomorphic to $H_{q}\left(S^{2 q+2} \mid F\right)$ which is dual to $H_{q+1}(F)$ (Lemma 1.3).

Definitions. The integer $r=\operatorname{rank} H_{q}(F)=\operatorname{rank} H_{q+1}(F)=\operatorname{rank} H_{q}(\tilde{X})$ depends on $K$ only and is called the rank of the free fibred knot $K$.

Denote by $M_{r}(R)$ the set of $r \times r$ matrices over a ring $R$. A Seifert triple is a triple of matrices $\left(A_{+} ; A_{-} ; B\right)$ where $A_{+}, A_{-} \in M_{r}(\mathbb{Z})$ and $B \in M_{r}\left(\mathbb{F}_{2}\right)$. Such a triple is called unimodular if $A_{+}$and $A_{-}$are unimodular. Two triples $\left(A_{+} ; A_{-} ; B\right)$ and $\left(A_{+}^{\prime} ; A_{-}^{\prime} ; B^{\prime}\right)$ are equivalent if there exist $X, Z \in G L_{r}(\mathbb{Z})$ and $Y \in M_{r}\left(\mathbb{F}_{2}\right)$ such that:

(a) $X^{T} A_{+} Z=A_{+}^{\prime}$ and $X^{T} A_{-} Z=A_{-}^{\prime}$,

(b) $\left(\begin{array}{cc}X & Y \\ 0 & Z\end{array}\right)^{T}\left(\begin{array}{ll}0 & A_{+} \\ A_{-}^{T} & B\end{array}\right)\left(\begin{array}{ll}X & Y \\ 0 & Z\end{array}\right)=\left(\begin{array}{cc}0 & A_{+}^{\prime} \\ \left(A_{-}^{\prime}\right)^{T} & B^{\prime}\end{array}\right)$ over $\mathbb{F}_{2}$.

This is clearly an equivalence relation.

Theorem 2-1. (Classification theorem). For $q \geqslant 4$, there is a one-to-one correspondence between:

(i) isotopy classes of $(q-1)$-connected $(2 q+1)$-submanifolds of $S^{2 q+2}$ such that: $H_{q}(F)$ is free abelian, $K=\partial F$ is non-empty and $(q-2)$-connected, $K$ is fibred and $F$ is a fibre-surface for $K$;

(ii) isotopy classes of (2q)-dimensional free fibred knots;

(iii) equivalence classes of unimodular Seifert triples.

The correspondence associates to a representative $F$ of the isotopy class in (i): the isotopy class of $\partial F$ for (ii); the equivalence class of the Seifert triple $\left(A_{+} ; A_{-} ; B\right)$ constructed as follows: Choose bases $\left\{\alpha_{k}\right\}$ for $H_{q}(F),\left\{\beta_{l}\right\}$ for $H_{q+1}(F)$ and lifts $\beta_{l}^{\#}$ in $\pi_{q+1}\left(F^{\prime}\right)$ and set $\left(A_{+}\right)_{i j}=A_{+}\left(\alpha_{i} ; \beta_{j}\right),\left(A_{-}\right)_{i j}=A_{-}\left(\alpha_{i} ; \beta_{j}\right), B_{i j}=\mathscr{A}\left(\beta_{i}^{\#} ; \beta_{j}^{\#}\right)$.

We decompose the proof of this theorem in a series of lemmas.

LEMMA 2.2. Let $F$ be the fibre-surface for a free fibred knot in $S^{2 q+2}$ and let $\left\{\alpha_{k}\right\},\left\{\beta_{k}\right\}$ and $\left\{\alpha_{k}^{\prime}\right\},\left\{\beta_{k}^{\prime}\right\}, k=1, \ldots, r$ be two bases of $H_{q}(F)$ and $H_{q+1}(F)$ respectively with lifts $\beta_{k}^{\#}$ and $\beta_{k}^{\prime \prime \#}$ in $\pi_{q+1}(F)$. Then the associated Seifert triples are equivalent.

Proof. Let $X$ (resp. $Z$ ) be the unimodular matrices expressing $\alpha_{k}^{\prime}$ (resp. $\beta_{k}^{\prime}$ ) in terms 
of $\alpha_{k}$ (resp. $\beta_{k}$ ). The matrix expressing the basis $\left\{\alpha_{k}^{\prime \prime \#} \beta_{k}^{\prime \#}\right\}$ of $\pi_{q+1}(F) \otimes \mathbb{F}_{2}$ in terms of

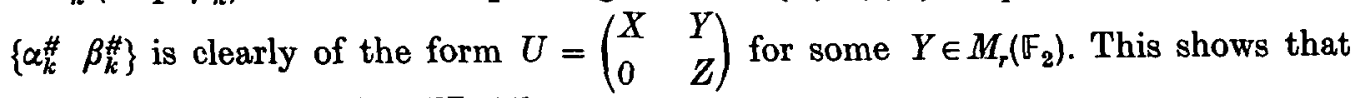
$A_{ \pm}^{\prime}=X^{T} A_{ \pm} Z$ and $\mathscr{A}^{\prime}=U^{T} \mathscr{A} U$.

LeMMa 2-3. Two isotopic free surfaces of a free knot have equivalent Seifert triples.

Proof. The isotopy induces isomorphisms between the homology and homotopy groups of the two surfaces. The Seifert triples computed with respect to corresponding bases will be the same.

The proof of theorem 3 of [7] applies without change to the following lemma:

LEMMA 2.4. Let $K$ be a free knot and let $V$ and $V^{\prime}$ be two free Seifert surfaces for $K$ with equivalent Seifert triples, then $V$ and $V^{\prime}$ are isotopic.

Lemma 2.5. Let $K$ be a 2q-dimensional free fibred knot, and let $F$ and $F^{\prime}$ be two fibresurfaces for $K$. Then $F$ and $F^{\prime}$ are isotopic.

Proof. Unfortunately the argument given in ([7], lemma 4) breaks down if the intersection forms of $F$ and $F^{\prime}$ are degenerate. One must instead consider the homology and homotopy Blanchfield pairings of $K$ (cf. [3]):

$$
\begin{aligned}
& \langle;\rangle: H_{q}(\tilde{X}) \times H_{q+1}(\tilde{X}) \rightarrow \mathbb{Q}(t) / \mathbb{Z}\left[t ; t^{-1}\right], \\
& {[;]: \pi_{q+1}(\tilde{X}) \otimes \mathbb{F}_{2} \times \pi_{q+1}(\tilde{X}) \otimes \mathbb{F}_{2} \rightarrow \mathbb{F}_{2}(t) / \mathbb{F}_{2}\left[t ; t^{-1}\right] .}
\end{aligned}
$$

These pairings can be computed using the surfaces $F$ and $F^{\prime}$ (cf. [3], §11): If $\left(A_{+} ; A_{-} ; B\right)$ and $\left(A_{+}^{\prime} ; A_{-}^{\prime} ; B^{\prime}\right)$ are the corresponding Seifert triples, then: $(t-1)\left(t A_{+}-A_{-}\right)^{-1}$ and $(t-1)\left(t A_{+}^{\prime}-A_{-}^{\prime}\right)^{-1}$ are matrix representatives of $\langle;\rangle$;

$$
(t-1)\left(t \mathscr{A}-\mathscr{A}^{T}\right)^{-1} \text { and }(t-1)\left(t \mathscr{A}^{\prime}-\mathscr{A}^{\prime} T\right)^{-1}
$$

are matrix representatives of $[;]$.

These pairings depend on $K$ only. Viewing $H_{k}(\tilde{X}) k=q, q+1$ as a free abelian group and $\pi_{q+1}(\tilde{X}) \otimes \mathbb{F}_{2}$ as a finite dimensional $\mathbb{F}_{2}$-vector space we see that there must exist unimodular matrices $X$ and $Z$ and a $\mathbb{F}_{2}$-matrix $Y$ such that:

$$
\begin{aligned}
& (t-1) Z^{-1}\left(t A_{+}-A_{-}\right)^{-1} X^{-T}=(t-1)\left(t A_{+}^{\prime}-A_{-}^{\prime}\right)^{-1} \quad \text { over } \mathbb{Q}\left[t ; t^{-1}\right] / \mathbb{Z}\left[t ; t^{-1}\right] \\
& (t-1)\left(\begin{array}{ll}
X & Y \\
0 & Z
\end{array}\right)^{-1}\left(t \mathscr{A}-\mathscr{A}^{T}\right)^{-1}\left(\begin{array}{ll}
X & Y \\
0 & Z
\end{array}\right)^{-T}=(t-1)\left(t \mathscr{A}^{\prime}-\mathscr{A}^{\prime}\right)^{-1} \quad \text { over } \mathbb{F}_{2}(t) / \mathbb{F}_{2}\left[t ; t^{-1}\right] .
\end{aligned}
$$

Set $\Delta(t)=\operatorname{det}\left(t A_{+}-A_{-}\right)$. These equations are in fact valid over $\mathbb{Z}\left[t ; t^{-1}\right] /(\Delta)$ and $\mathbb{F}_{2}\left[t ; t^{-1}\right] /\left(\Delta^{2}\right)$ respectively. Since $\Delta$ is a monic polynomial, we can apply the arguments of [(9], proposition 2) to conclude that $\left(A_{+} ; A_{-} ; B\right)$ and $\left(A_{+}^{\prime} ; A_{-}^{\prime} ; B^{\prime}\right)$ are equivalent Seifert triples. Lemma 2.4 shows that $F$ and $F^{\prime}$ are isotopic.

To prove the realization part of the theorem we need some of the information given in the following lemma, the full content of which will be used in the next section.

Lемма 2*6. Let $V$ be a free surface for a $2 q$-dimensional knot and let $k$ be an integer. Then for all $\left(u^{\#} ; v^{\#}\right) \in \pi_{q}(V ; \partial V) \times \pi_{q+1}(V ; \partial V)$ there are proper embeddings

$$
f_{u}:\left(D^{q} ; \partial D^{q}\right) \hookrightarrow(V ; \partial V) \text { and } f_{v}:\left(D^{q+1} ; \partial D^{q+1}\right) \hookrightarrow(V ; \partial V)
$$




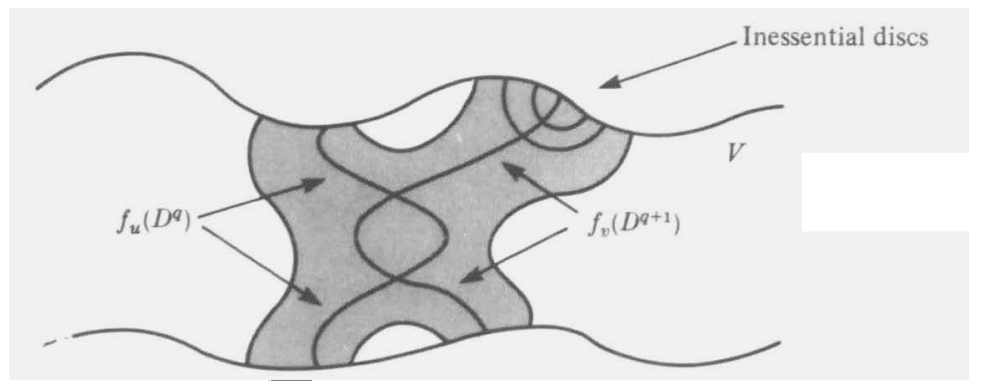

Fig. 1

and an embedding $g: D^{2 q+1} \hookrightarrow V$ such that: $f_{u}\left(D^{q}\right)$ and $f_{v}\left(D^{q+1}\right)$ intersect in $V$ in exactly $k$ points, $f_{u}\left(D^{q}\right)$ and $f_{v}\left(D^{q}\right)$ are contained ing $\left(D^{2 q+1}\right), \partial V \cap g\left(D^{2 q+1}\right)$ is a tubularneighbourhood of $f_{u}\left(\partial D^{q}\right) \cup f_{v}\left(\partial D^{q+1}\right)$ (ef. Fig. 1).

Proof. Since $q \geqslant 4$ and $(V ; \partial V)$ is a $(q-1)$-connected pair, we may represent $u$ and $v$ by proper embeddings $f_{u}^{\prime}$ and $f_{v}^{\prime}$ intersecting in a finite number of points (say $l$ ). Construct $l-1$ properly embedded $q$-dimensional discs that intersect each $f_{v}^{\prime}\left(D^{q+1}\right)$ in 1 oriented point in $\dot{V}^{\circ}$ and compress to $\partial V$ (cf. Fig. 1). Tube these dises together and to $f_{u}^{\prime}\left(D^{q}\right)$ inside $\partial V$ to produce a properly embedded dise still representing $u$. Apply the Whitney trick to reduce the geometric intersection number to 1 . Let $f_{u}^{n}$ and $f_{v}^{\prime \prime}$ denote the new embeddings and let $T_{u}$ and $T_{v}$ be their tubular neighbourhoods. $T_{u} \cup T_{v}$ is an embedded $(2 q+1)$-dise with corners. Construct again $k-1 q$-dimensional dises intersecting $f_{v}^{\prime \prime}$ in one oriented point inside $T_{v}$ which compress to $\partial V$ and tube them together; choose two arcs with the same end points connecting these discs to $f_{u}^{\prime \prime}\left(\partial D^{q}\right)$ one of which is in $\partial V$ and the other in $T_{u} \cup T_{v}$. The union of these two arcs bounds a 2-disc which can be fattened to a $(2 q+1)$-dimensional disc $\Delta$. The union of $\Delta$ and $T_{u} \cup T_{v}$ is again a $(2 q+1)$-disc inside which Whitney trick can be applied to get the desired result.

Lemma 2.7. For each Seifert triple $\left(A_{+} ; A_{-} ; B\right)$ and each $q \geqslant 4$ there exists a free Seifert surface $V$ in $S^{2 q+2}$ which realizes $\left(A_{+} ; A_{-} ; B\right)$.

Proof. (By induction on the dimension $d$ of the matrices). For $d=0, V$ is a $(2 q+1)$ dimensional disc. If $d \geqslant 1$ let $V_{0}$ be a free Seifert surface realizing $\left(A_{+}^{0}, A_{-}^{0} ; B^{0}\right)$ where $X^{0}$ denotes the upper left $d \times d$ corner of the matrix $X$. Set

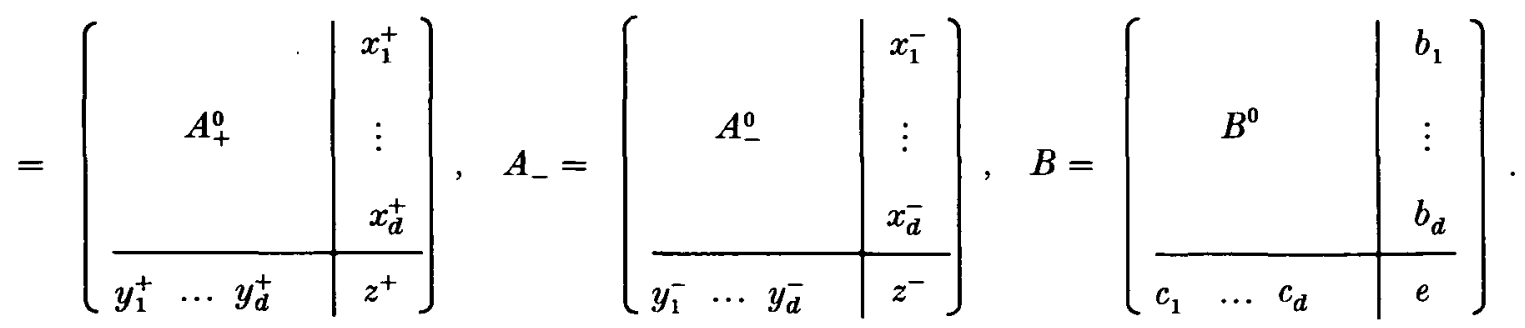

Let $\left\{\alpha_{i}\right\}\left(\operatorname{resp} .\left\{\beta_{i}\right\}\right)$ be a basis of $H_{q}\left(V_{0}\right)$ (resp. $\left.H_{q+1}\left(V_{0}\right)\right), i=1, \ldots, d$, with lifts $\beta_{i}^{\#} \in \pi_{q+1}\left(V_{0}\right)$. Since

and

$$
J: H_{q}\left(V_{0}\right) \times H_{q+1}\left(V_{0} ; \partial V_{0}\right) \rightarrow \mathbb{Z}
$$$$
J^{\prime}: H_{q}\left(V_{0} ; \partial V_{0}\right) \times H_{q+1}\left(V_{0}\right) \rightarrow \mathbb{Z}
$$ 
are unimodular and $\mathscr{J}: \pi_{q+1}\left(V_{0}\right) \otimes \mathbb{F}_{2} \times \pi_{q+1}\left(V_{0} ; \partial V_{0}\right) \otimes \mathbb{F}_{2} \rightarrow \mathbb{F}_{2}$ is non-singular (Lemma $1 \cdot 10)$, there exist $u \in H_{q}\left(V_{0} ; \partial V_{0}\right)$ and $v^{\#} \in \pi_{q+1}\left(V_{0} ; \partial V_{0}\right)$ such that

and

$$
\begin{aligned}
J\left(\alpha_{i} ; h v^{\#}\right) & =x_{i}^{+}-x_{i}, \\
J^{\prime}\left(u ; \beta_{i}\right) & =y_{i}^{+}-y_{\bar{i}}^{-},
\end{aligned}
$$

By Lemma $2 \cdot 6$ we can represent $u$ and $v^{\#}$ by proper embeddings $f_{u}: D^{a} \hookrightarrow V_{0}$, $f_{v}: D^{q+1} \hookrightarrow V_{0}$, such that $f_{u}$ and $f_{v}$ intersect in $z_{+}-z_{-}$points. Let $g_{u}: D^{q} \hookrightarrow S^{2 q+2} \mid V_{0}$ be an embedding such that $\partial V_{0} \cap g_{u}\left(D^{q}\right)=g_{u}\left(\partial D^{q}\right)=f_{u}\left(\partial D^{q}\right)$. Thicken $g_{u}\left(D^{q}\right)$ to a $q$-handle of dimension $2 q+1$ meeting $V_{0}$ in a tubular neighbourhood of $f_{u}\left(\partial D^{q}\right)$ and denote by $V^{\prime}$ the union of $V_{0}$ and this handle. Note that $i_{ \pm}: V_{0} \rightarrow S^{2 q+2} \mid V_{0}$ extend to maps $i_{ \pm}: V^{\prime} \rightarrow S^{2 q+2} \mid V^{\prime}$. We can find an embedding $g_{v}: D^{a+1} \hookrightarrow S^{2 q+2} \mid V^{\prime}$ such that:

$$
\begin{aligned}
& \partial V^{\prime} \cap g_{v}\left(D^{q+1}\right)=g_{v}\left(\partial D^{q+1}\right)=f_{v}\left(\partial D^{q+1}\right), \\
& L\left(S_{u} ; i_{+} \beta_{i}\right)=y_{i}^{+}, \\
& L\left(i_{-} \alpha_{i} ; S_{v}\right)=x_{i}^{+}, \\
& \mathscr{L}\left(i_{-} \beta_{i}^{\#} ; S_{v}\right)=b_{i}, \\
& L\left(S_{u} ; i_{+} S_{v}\right)=z^{+},
\end{aligned}
$$

where

$$
S_{u}=g_{u}\left(D^{q}\right) \cup f_{u}\left(D^{q}\right), \quad S_{v}=g_{v}\left(D^{q+1}\right) \cup f_{v}\left(D^{q+1}\right) .
$$

Consider a tubular neighbourhood $\tau_{v}$ of $S_{v}$ in $S^{2 q+2}: \tau_{v}$ is a trivial $(q+1)$-disc bundle over $S^{q+1}$ and $i_{+} \mid f_{v}\left(D^{q+1}\right)$ is a section of the associated sphere bundle over $f_{v}\left(D^{a+1}\right)$. The extensions of this section to a section over $S_{v}$ are in one-to-one correspondence with elements of $\pi_{q+1}\left(S^{q}\right) \simeq \mathbb{Z} / 2$. Consider the section corresponding to $e \in \mathbb{F}_{2} ;$ the orthogonal complement to this section gives a $(q+1)$-handle attached to $V^{\prime}$. Let $V$ be the union of $V^{\prime}$ and this handle. Lemma $1 \cdot 10$ shows that $V$ has Seifert triple $\left(A_{+} ; A_{-} ; B\right)$.

The proof of Theorem $2 \cdot 1$ is straightforward from the preceding lemmas.

Using Theorem $2 \cdot 1$, it is easy to classify free fibred $(2 q)$-dimensional knots of rank 1 .

LEMMA 2-8. There are exactly 4 types of free fibred knots of rank 1, corresponding to the equivalence classes of Seifert triples

$$
\begin{aligned}
& (1 ; 1 ; 0) \sim(-1 ;-1 ; 0) \\
& (1 ; 1 ; 1) \sim(-1 ;-1 ; 1) \\
& (1 ;-1 ; 0) \sim(-1 ; 1 ; 0) \\
& (1 ;-1 ; 1) \sim(-1 ; 1 ; 1) .
\end{aligned}
$$

Definition. In analogy with [10], §2, we call these knots Hopf knots and their fibresurfaces Hopf bands.

\section{Plumbing}

Let $Y$ be a disjoint union of copies of $S^{q} \times D^{q}$ and $S^{q-1} \times D^{q+1}$ and let $f: Y_{\hookrightarrow} \partial D^{2 q+1}$ be an embedding.

Let $K_{1}$ and $K_{2}$ be two free fibred knots bounding fibre-surfaces $F_{1}$ and $F_{2}$ in $S^{2 q+2}$. Divide $S^{2 q+2}$ into two hemispheres $B_{1}$ and $B_{2}$ intersecting in a $(2 q+1)$-dimensional sphere $S$. Let $\psi: D^{2 q+1} \hookrightarrow S$ be an embedding and suppose that: 
(i) $F_{i} \subseteq B_{i} \quad(i=1,2)$,

(ii) $F_{1} \cap S=F_{2} \cap S=F_{1} \cap F_{2}=\psi\left(D^{2 q+1}\right)$,

(iii) $\partial F_{1} \cap \psi\left(D^{2 q+1}\right)=F_{2} \cap \psi\left(\partial D^{2 q+1}\right)=\psi \circ f(Y)$ $\partial F_{2} \cap \psi\left(D^{2 q+1}\right)=F_{1} \cap \psi\left(\partial D^{2 q+1}\right)=\overline{\psi\left(\partial D^{2 q+1}\right) \backslash \psi \circ f(Y)}$,

(iv) the orientations of $F_{1}$ and $F_{2}$ match on $\psi\left(D^{2 q+1}\right)$.

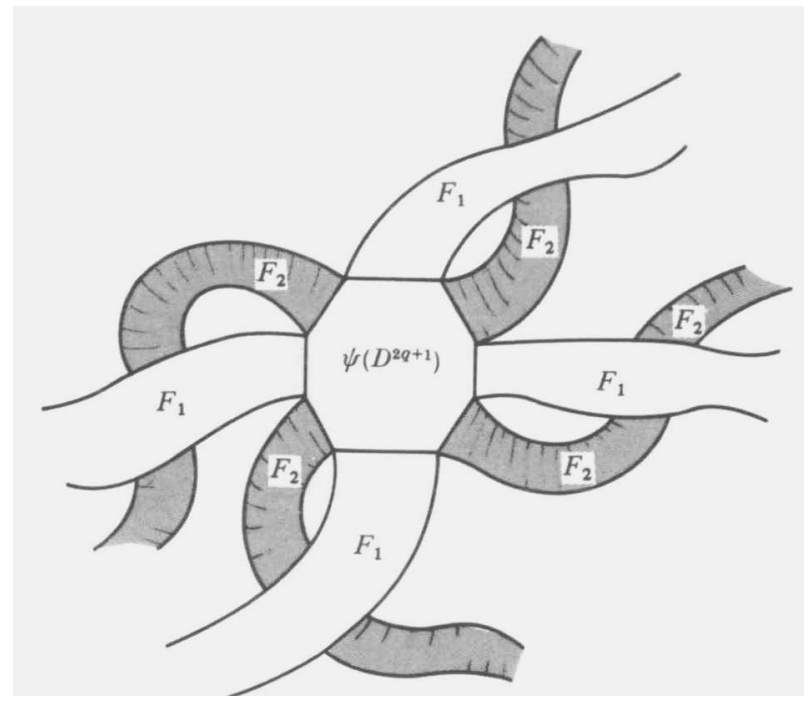

Fig. 2

Fig. 2 illustrates the situation. This construction is reminiscent of the generalized plumbing for classical knots considered in [11], $\$ 2$.

LEMMA 3·1. $F_{1} \cup F_{2}$ is a manifold with corners.

Proof. Consider a collar $C$ of $\psi\left(D^{2 q+1}\right)$ in $F_{2}: G_{1}=F_{1} \cup C$ is clearly a manifold with corners. There is an embedding $\phi: Y \times[0,1] \hookrightarrow F_{2}$ such that $\phi(Y \times\{0\})=f(Y) ; F_{2}$ cut along $\phi(Y \times(0,1))$ is a manifold with corners consisting of two components one of which is $\psi\left(D^{2 q+1}\right)$, denote by $G_{2}$ the other component. $F_{1} \cup F_{2}$ can be viewed as $G_{1} \cup G_{2}$ sewn along $\phi(Y \times\{1\})$ and is therefore a manifold with corners.

Denote by $F_{1} \square F_{2}$ the submanifold of $S^{2 q+2}$ obtained by smoothing the corners of $F_{1} \cup F_{2}\left(F_{1} \square F_{2}\right.$ depends of course on $\psi, f$ and the decomposition of $\left.S^{2 q+2}\right)$.

Definition. $F_{1} \square F_{2}$ is said to be obtained by plumbing together $F_{1}$ and $F_{2}$.

Proposition 3.2. If $K_{1}$ and $K_{2}$ are free fibred knots with fibre-surfaces $F_{1}$ and $F_{2}$, $K=\partial\left(F_{1} \square F_{2}\right)$ is a free fibred knot with fibre-surface $F_{1} \square F_{2}$. If $\left(A_{+}^{i} ; A_{-}^{i} ; B^{i}\right) i=1,2$ are Seifert triples for $F_{i}, F_{1} \square F_{2}$ admits the Seifert triple $\left(A_{+} ; A_{-} ; B\right)$ where either:

or

$$
\begin{array}{lll}
\left(^{*}\right) A_{+}=\left(\begin{array}{ll}
A_{+}^{1} & C_{+} \\
0 & A_{+}^{2}
\end{array}\right), & A_{-}=\left(\begin{array}{ll}
A_{-}^{1} & 0 \\
C_{-} & A_{-}^{2}
\end{array}\right), & B=\left(\begin{array}{ll}
B^{1} & D \\
0 & B^{2}
\end{array}\right) \\
\left(^{* *}\right) A_{+}=\left(\begin{array}{ll}
A_{+}^{1} & 0 \\
C_{+} & A_{+}^{2}
\end{array}\right), & A_{-}=\left(\begin{array}{ll}
A_{-}^{1} & C_{-} \\
0 & A_{-}^{2}
\end{array}\right), & B=\left(\begin{array}{ll}
B^{1} & 0 \\
D & B^{2}
\end{array}\right)
\end{array}
$$

for some integral matrices $C_{+}, C_{-}$and some $\mathbb{F}_{2}$-matrix $D$. 
Proof. Van Kampen and Mayer-Vietoris arguments show that $K$ is $(q-2)$-connected; $F=F_{1} \square F_{2}$ is clearly $(q=1)$-connected and

$$
\begin{gathered}
H_{k}(F) \simeq H_{k}\left(F_{1}\right) \oplus H_{k}\left(F_{2}\right) \quad(k=1, q+1), \\
\pi_{q+1}(F) \simeq \pi_{q+1}\left(F_{1}\right) \oplus \pi_{q+1}\left(F_{2}\right) \quad \text { (using Whitehead's exact sequence) }
\end{gathered}
$$

So we may choose for $F$ the bases corresponding to the direct sum decomposition and represent these by embeddings in the relevant $F_{i}$. Suppose that $i_{+}: F \rightarrow S^{2 q+2} \backslash F$ pushes all points of $\psi\left(D^{2 q+1}\right)$ into $\dot{B}_{1}$. The Seifert triple has the form given in $\left(^{*}\right)$ since $i_{+}\left(F_{1}\right)$ is separated from $F_{2}$ by a small push-off of the sphere $S$. Similarly if $i_{+}$pushes $\psi\left(D^{2 q+1}\right)$ into $\stackrel{\circ}{B}_{2},\left(^{* *}\right)$ holds. Since $\operatorname{det} A_{ \pm}=\operatorname{det} A_{ \pm}^{1} \operatorname{det} A_{ \pm}^{2}$, Proposition 1.6 shows that $K$ is a fibred knot with fibre-surface $F$.

Definition. A (2q)-dimensional knot $K$ is said to be obtained by plumbing if there is a sequence of $(2 q+1)$-manifolds $F_{0}, F_{1}, \ldots, F_{s}$ embedded in $S^{2 q+2}$ such that $F_{0}=D^{2 q+1}$, $\partial F_{s}=K$ and $F_{i+1}$ is obtained by plumbing together $F_{i}$ and a Hopf band.

Remark. $K$ is a free fibred knot by Proposition $3 \cdot 2$.

Definition. A Seifert triple $\left(T_{+} ; T_{-} ; B\right)$ is called triangular if $T_{+}$(resp. $T_{-}$) is unimodular and upper (resp. lower) triangular.

Lemma 3-3. Any triangular triple $\left(T_{+} ; T_{-} ; B\right)$ is equivalent to $\left(T_{+} ; T_{-} ; \bar{B}\right)$ where $\bar{B}$ is upper triangular.

Proof. Since $T_{+}$and $T_{-}$are triangular and have \pm 1 in their diagonal, one can change by row and colum operations $B$ to an upper triangular matrix $\bar{B}$ without affecting $T_{+}$and $T_{-}$.

THEOREM 3.4. A free fibred knot is obtained by plumbing if and only if it admits a triangular Seifert triple.

Proof. Let $K$ be obtained by plumbing. We prove the theorem by induction on the rank $r$ of $K$. If $r=1, K$ is a Hopf knot. Let $F^{\prime}$ be the fibre-surface obtained by plumbing together the $r$ first Hopf bands. By induction $F^{\prime}$ admits a triangular triple $\left(T_{+} ; T_{-} ; B^{\prime}\right)$ Proposition $3 \cdot 2$ shows that plumbing a Hopf band of type $\left(\epsilon_{+} ; \epsilon_{-} ; b\right)$ together with $F^{\prime}$ produces a free Seifert surface with triple $\left(A_{+} ; A_{-} ; B\right)$, where

$$
\begin{aligned}
& A_{+}=\left(\begin{array}{ccc|c} 
& & x_{+} & \vdots \\
& & T_{+} & x_{r} \\
\hline 0 & \ldots & 0 & \epsilon_{+}
\end{array}\right), \quad A_{-}=\left(\begin{array}{ccc|c} 
& & 0 \\
& & \vdots \\
& & 0 \\
\hline y_{1} & \ldots & y_{r} & \epsilon_{-}
\end{array}\right)
\end{aligned}
$$

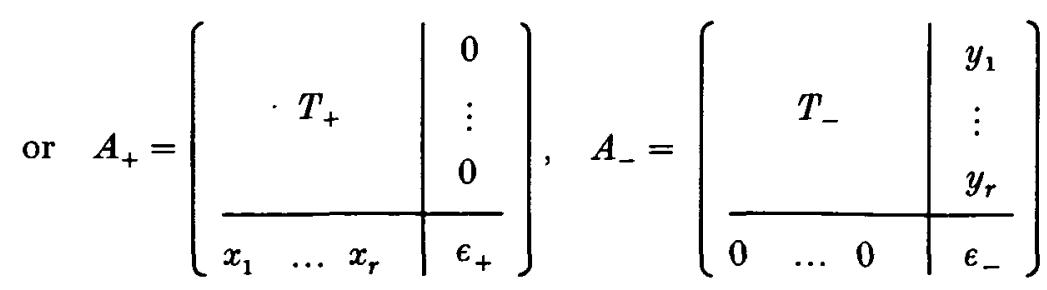


In the second case perform column operations using $\epsilon_{+}$to annihilate $\left(x_{1} \ldots x_{r}\right)$ and row operations using $\epsilon_{-}$to annihilate $\left(y_{1} \ldots y_{r}\right)^{T}$; this changes $A_{+}$(resp. $A_{-}$) to an upper (resp. lower) triangular matrix, so that $\left(A_{+} ; A_{-} ; B\right)$ is equivalent to a triangular triple.

Conversely suppose that $K$ admits a triangular triple $\left(T_{+} ; T_{-} ; B\right)$. We may assume that $B$ is upper triangular (Lemma 3.3) and we perform an induction on the dimension $d$ of the matrices. If $d=1$, choose the Hopf band corresponding to the triple. If $d \geqslant 2\left(T_{+} ; T_{-} ; B\right)$ is of the form

$T_{+}=\left(\begin{array}{ccc|c} & & x_{1} \\ & T_{+}^{\prime} & \vdots \\ & & x_{d} \\ \hline 0 & \ldots & 0 & \epsilon_{+}\end{array}\right), \quad T_{-}=\left(\begin{array}{ccc|c}T_{-}^{\prime} & \vdots \\ & & 0 \\ \hline y_{1} & \ldots & y_{d} & \epsilon_{-}\end{array}\right), B=\left(\begin{array}{ccc|c} & & b_{1} \\ & & \vdots \\ & & b_{d} \\ \hline 0 & \ldots & 0 & b_{0}\end{array}\right)$.

By induction there is a Seifert surface obtained by plumbing Hopf bands admitting the triangular triple $\left(T_{+}^{\prime} ; T_{-}^{\prime} ; B^{\prime}\right)$ with respect to some bases $\left\{\alpha_{i}\right\},\left\{\beta_{i}\right\}$ and lifts $\beta_{i}^{H}$, $i=1, \ldots, d$. Lemma $2 \cdot 6$ and the same argument as in the proof of Lemma $2 \cdot 7$ show that there are proper embeddings $f_{u}: D^{q} \hookrightarrow F^{\prime}, f_{v}: D^{q+1} \hookrightarrow F^{\prime}$ intersecting in $\epsilon_{+}-\epsilon_{-}^{r}$ points representing homotopy classes $u^{\#}$ and $v^{\#}$ such that

$$
\begin{aligned}
J\left(\alpha_{i} ; h v^{\#}\right) & =x_{i}, \\
J^{\prime}\left(h u^{\#} ; \beta_{i}\right) & =y_{i}, \\
\mathscr{J}\left(\beta_{i}^{\#} ; v^{\#}\right) & =b_{i} .
\end{aligned}
$$

Furthermore, by Lemma $2 \cdot 6$ the image of these embeddings is contained in a $(2 q+1)$ disc $\Delta$ embedded in $F^{\prime}$ meeting $\partial F^{\prime}$ in a tubular neighbourhood of $f_{u}\left(\partial D^{q}\right)$ and $f_{v}\left(\partial D^{q+1}\right)$. Thicken $\Delta$ on the negative side of $F^{\prime}$ to get a $(2 q+2)$-ball $B$; inside this ball attach a $q$-handle and a $(q+1)$-handle as described in the proof of Lemma 2.7. The new surface $F$ admits $\left(T_{+} ; T_{-} ; B\right)$ as a Seifert triple. The union of $\Delta$ and the two attached handles forms a Hopf band of type $\left(\epsilon_{+} ; \epsilon_{-} ; b_{0}\right)$ which is plumbed together with $F^{\prime}$. The classification theorem shows that $\partial F$ is isotopic to $K$.

Examples of spherical free fibred knots not obtained by plumbing

Lemma 3.5. Let $p$ be a prime, $n$ a positive integer and $\epsilon_{i}= \pm 1$ for $i=1,2$. To any solution of

$$
\left(^{*}\right)\left\{\begin{array}{l}
x^{n} \equiv \epsilon_{1}\left(p^{n}\right) \\
(x+1)^{n} \equiv \epsilon_{2}\left(p^{n}\right) \\
2 x+1 \not \equiv 0(p)
\end{array}\right.
$$

there correspond Seifert triples that are not equivalent to any triangular triple.

Proof. Let $a$ be a solution of $\left({ }^{*}\right)$ and let $\alpha$ and $\beta$ be integers such that $a^{n}=\epsilon_{1}+\alpha p^{n}$, $(a+1)^{n}=\epsilon_{2}+\beta p^{n}$. Set 


$$
\begin{aligned}
& A_{+}=\left(\begin{array}{ccccccc}
a+1 & p & & & \\
0 & a+1 & & p & & \\
\vdots & & \ddots & \ddots & \ddots & p \\
0 & \bigcirc & & \ddots & & p \\
(-1)^{n} \alpha p & 0 & \ldots & 0 & a+1
\end{array}\right) \\
& A_{-}=\left(\begin{array}{ccccc}
a & p & & & \\
0 & a & & p & \\
\vdots & & \ddots & & \\
0 & \bigcirc & & p \\
(-1)^{n} \beta p & 0 & \ldots & 0 & a
\end{array}\right)
\end{aligned}
$$

and let $B$ be any $\mathbb{F}_{2}$-matrix. $\operatorname{det} A_{+}=\epsilon_{1}$, $\operatorname{det} A_{-}=\epsilon_{2}$ and $\operatorname{det}\left(A_{+}-A_{-}\right)=1$. If $\left(A_{+} ; A_{-} ; B\right)$ were equivalent to a triangular triple $\left(T_{+} ; T_{-} ; B^{\prime}\right)$ there would exist unimodular matrices $X$ and $Z$ such that $X^{T}\left(A_{+}-t A_{-}\right) Z=T_{+}-t T_{-}$over $\mathbb{Z}[t]$. Project this equality to $\mathbb{F}_{p}[t]$ and denote by $\mathfrak{a}$ the ideal of $\mathbb{F}_{p}[t]$ generated by the 1-minors of $A_{+}-t A_{-}$; we have $a=((a+1)-t a)$. On the other hand, since $T_{+}$and $T_{-}$have \pm 1 on their diagonal entries, $\mathfrak{a}$ should contain $1-t$ or $1+t$ (or both). The only possibility is that $2 a+1 \equiv 0(p)$ which contradicts $\left({ }^{*}\right)$.

\section{Examples of solutions of $\left({ }^{*}\right)$}

Set $n=3, p=7, \epsilon_{1}=-1, \epsilon_{2}=+1 ; a=18$ is a solution of $\left(^{*}\right)$ with $\alpha=20, \beta=17$.

For $n=p-1, \epsilon_{1}=\epsilon_{2}=1$, the following values are taken from $[1]$, p. $68\left(\left[a_{0}, a_{1}, \ldots\right.\right.$, $\left.a_{p-1}\right]$ denotes $\left.\sum_{i=0}^{p-1} a_{i} p^{i}\right)$ :

$$
\begin{aligned}
& p=7: \quad a=[2,4,6,3,0,2] \text { and } a=[4,2,0,3,6,4] \\
& p=13 ; a=[3,11,6,9,7,2,4,4,8,8,1,5]
\end{aligned}
$$

and

$$
a=[9,1,6,3,5,10,8,8,4,4,1,11,7] \text {. }
$$

TheOREM 3.6. For any $q \geqslant 4$ there exist spherical $(2 q)$-dimensional free fibred knots of arbitrarily high rank that are not obtained by plumbing.

Proof. Consider Seifert triples obtained by taking block sums of the matrices described in Lemma 3.5 and realize them by free fibred knots.

\section{Applications to spinning}

In this section we consider odd-dimensional knots as defined in [10]. Recall that one can give the following definition for the spinning of a knot (cf. [4]):

Let $K$ be a (not necessarily spherical) $(2 q-1)$-dimensional knot and $B_{0}$ be a $(2 q+1)$ dimensional ball in $S^{2 q+1}$ such that $B_{0} \cap K$ is a $(2 q-1)$-ball and the pair $\left(B_{0} ; B_{0} \cap K\right)$ is unknotted. Set $B=S^{2 q+1} \backslash \stackrel{\circ}{0}_{0}$, and $\beta=K \backslash \stackrel{\circ}{0}_{0}$. 
Definition. The spinning of $K$ is the (2q)-dimensional knot

$$
\sigma(K)=\left(\partial \beta \times D^{2}\right) \cup_{\partial}\left(\beta \times \partial D^{2}\right)
$$

in the sphere $S^{2 q+2}=\left(\partial B \times D^{2}\right) \cup\left(B \times \partial D^{2}\right)$.

Let $V$ be a Seifert surface for $K$, and suppose that the pair $\left(B_{0} ; B_{0} \cap V\right)$ is diffeomorphic to the pair $\left(D^{2 q+1} ; H\right)$ where $D^{2 q+1}$ denotes the unit disc in $\mathbb{R}^{2 q+1}$ and

$$
H=\left\{\left(x_{1}, \ldots, x_{2 q+1}\right) \in D^{2 q+1} \mid x_{2 q} \geqslant 0, x_{2 q+1}=0\right\} .
$$

Set $V^{\prime}=V \cap B$ and $\Delta=V \cap \partial B$; then

$$
\sigma(V)=\left(\Delta \times D^{2}\right) U_{\Delta \times \partial D^{2}}\left(V^{\prime} \times \partial D^{2}\right)
$$

is a Seifert surface for $\sigma(K)$ in $S^{2 q+2}$.

THEOREM 4.1. Let $K$ be a simple $(2 q-1)$-dimensional knot, and suppose that $q \geqslant 4$.Then

(i) $\sigma(K)$ is a free $(2 q)$-dimensional knot;

(ii) if $V$ is a simple Seifert surface for $K$ with associated Seifert matrix $A, \sigma(V)$ is a free Seifert surface for $\sigma(K)$ with associated Seifert triple $\left(A ;(-1)^{q+1} A^{T} ; 0\right)$;

(iii) $K$ is fibred with fibre-surface $F$ if and only if $\sigma(K)$ is a free fibred knot with fibresurface $\sigma(F)$;

(iv) if $K$ is obtained by plumbing, so is $\sigma(K)$.

Proof. We refer to [10] for the properties of odd-dimensional knots we use.

(i) Let $V$ be a simple Seifert surface for $K$. $\tilde{H}_{k}(V)=0$ for $k \neq q$ and $H_{q}(V)$ is free abelian. It is easy to see that $\sigma(V)$ is $(q-1)$-connected and that

$$
\begin{aligned}
H_{q}(\sigma(V)) & \simeq H_{q}(V) \otimes H_{0}\left(S^{1}\right) \simeq H_{q}(V), \\
H_{q+1}(\sigma(V)) & \simeq H_{q}(V) \otimes H_{1}\left(S^{1}\right) \simeq H_{q}(V) .
\end{aligned}
$$

This shows that $\sigma(V)$ is a free Seifert surface for $\sigma(K)$.

(ii) Let $\left(\gamma_{i}\right\}(i=1, \ldots, r)$ be a basis of $H_{q}(V)$; we may represent $\gamma_{i}$ by an embedding $\phi_{i}: S^{q} \hookrightarrow V^{\prime}$. The embeddings

$$
\begin{gathered}
a_{i}: S^{q} \hookrightarrow V^{\prime} \times \partial D^{2} \subseteq \sigma(V) \\
x \mapsto\left(\phi_{i}(x) ; 1\right)
\end{gathered}
$$

and

$$
\begin{gathered}
b_{j}: S^{a} \times S^{1} \hookrightarrow V^{\prime} \times \partial D^{2} \subseteq \sigma(V) \\
(x ; t) \mapsto\left(\phi_{i}(x) ; t\right)
\end{gathered}
$$

represent a basis of $H_{q}(\sigma(V))$ (resp. $H_{q+1}(\sigma(V))$.

Let $C_{i}$ be a singular $(q+1)$-chain in $B$ such that $\partial C_{i}=\phi_{i}\left(S^{q}\right)$; the algebraic intersection number of $C_{i}$ and $i_{+} \phi_{j}\left(S^{q}\right)$ is $L_{S 2 q+2}\left(\gamma_{i} ; i_{+} \gamma_{j}\right)$. The singular chain

$$
C_{i} \times\{1\} \subseteq B \times \partial D^{2} \subseteq S^{2 q+2}
$$

intersects algebraically $i_{+} b_{j}\left(S^{q} \times S^{1}\right)$ also in $L_{s^{2 q+1}}\left(\gamma_{i} ; i_{+} \gamma_{j}\right)$ points. As $a_{i}\left(S^{q}\right)$ bounds $C_{i} \times\{1\}$, this shows that with respect to these bases $A_{+}=A$. Similarly,

$$
L_{s^{2 q+1}}\left(\gamma_{i} ; i_{-} \gamma_{j}\right)=L_{s^{2 q+2}}\left(a_{i}\left(S^{q}\right) ; i_{-} b_{j}\left(S^{q} \times S^{1}\right)\right) \text {; }
$$

since

$$
L_{s 2 q+1}\left(\gamma_{i} ; i_{-} \gamma_{j}\right)=(-1)^{q+1} L_{s 2 q+1}\left(\gamma_{j} ; i_{+} \gamma_{i}\right)
$$

we have $A_{-}=(-1)^{q+1} A^{T}$. 
To compute the homotopy linking form, consider the submanifold $\bar{V}=V^{\prime} \times D^{2}$ of the $(2 q+3)$-dimensional ball $B \times D^{2}$. The normal bundle to $\bar{V}$ in $B \times D^{2}$ is trivial, so that $i_{+}: \sigma(V) \hookrightarrow S^{2 q+2}$ extends to a map $i_{+}: \bar{V} \hookrightarrow B \times D^{2} . \bar{V}$ has the homotopy type of $V$, so that $\pi_{q}(\bar{V}) \simeq H_{q}(\bar{V})$ and $H_{q+1}(\bar{V})=0$. Consider the two exact sequences:

$$
\begin{aligned}
& 0 \rightarrow \pi_{q}(\sigma(V)) / 2 \pi_{q}(\sigma(V)) \rightarrow \pi_{q+1}(\sigma(V)) \rightarrow H_{q+1}(\sigma(V)) \rightarrow 0 \\
& \begin{array}{l}
\stackrel{\downarrow}{\downarrow i_{1}} \\
\quad \downarrow \pi_{q}(\bar{V}) / 2 \pi_{q}(\bar{V}) \longrightarrow \pi_{q+1}(\bar{V}) \longrightarrow H_{q+1}(\bar{V})=0 \rightarrow 0,
\end{array}
\end{aligned}
$$

where $i_{1}$ and $i_{2}$ are induced by the inclusion of $\sigma(V)$ in $\bar{V}$. As $i_{1}: H_{q}(\sigma(V)) \rightarrow H_{q}(\bar{V})$ is an isomorphism, so is $i_{1}: \pi_{q}(\sigma(V)) \rightarrow \pi_{q}(\bar{V})$, and it is easy to see using $\left(^{*}\right)$ that any $u \in H_{q+1}(\sigma(V))$ admits a lift $u^{\#} \in \pi_{q+1}(\sigma(V))$ such that $i_{2}\left(u^{\#}\right)=0$. For $j=1, \ldots, r$, let $\bar{b}_{j}: S^{q+1} \hookrightarrow S^{2 q+2}$ be an embedding such that $h\left(\left[\bar{b}_{j}\right]\right)=b_{j}$ and $i_{2}\left(\bar{b}_{j}\right)=0 ; \bar{b}_{j}$ extends to a map $F_{j}: D^{q+2} \rightarrow \bar{V}$, and since $q \geqslant 4$ we may assume that $F_{j}$ is an embedding. By Lemma $1 \cdot 7 ; \mathscr{A}\left(\left[\bar{b}_{k}\right] ;\left[\bar{b}_{l}\right]\right)=\mathscr{L}_{S^{2 q+1}}\left(\bar{b}_{k} ; i_{+} \bar{b}_{l}\right)=0$ since $F_{k}\left(D^{q+2}\right) \cap i_{+} F_{l}\left(D^{q+2}\right)=\varnothing$.

(iii) $K$ is fibred if and only if $A$ is unimodular ([10], lemma $1 \cdot 1) ;\left(A ;(-1)^{q+1} A^{T} ; 0\right)$ is a unimodular triple if and only if $\sigma(K)$ is fibred with fibre-surface $\sigma(F)$ (Proposition 1.6).

(iv) If $K$ is obtained by plumbing, $K$ admits a unimodular lower (or upper) triangular Seifert matrix (cf. [10], proposition 2.4). This shows that $\left(A ;(-1)^{q+1} A^{T} ; 0\right)$ is a triangular triple and that $\sigma(K)$ is obtained by plumbing.

Remark. Part (ii) of this theorem is the analogue for Seifert triples of the result of C. Kearton [4] concerning the Blanchfield pairing of a simple spun knot.

I would like to thank C. Kearton and M. A. Armstrong for their help and encouragement during the preparation of this article. I also thank the Department of Mathematics of the University of Durham for their hospitality and the Science and Engineering Research Council of Great Britain for their grant.

\section{REFERENCES}

[1] W. Berwick. Integral bases. Cambridge Tracts 22. (Cambridge University Press, 1927).

[2] A. Haefuiger. Plongements différentiables de veriétés dans variétés. Comment. Math. Helv. 36 (1962), 47-82.

[3] C. Kearton. An algebraic classification of certain simple even dimensional knots. Trans. Amer. Math. Soc. 276 (1983), 1-53.

[4] C. Kearton. Simple spun knots. Topology 23 (1984), 91-95.

[5] M. Kervatre. Les noeuds de dimensions supérieures. Bull. Soc. Math. France 93 (1965), 225-271.

[6] M. Kervatre and C. Weber. A survey of multidimensional knots. Knot Theory (Proceedings, Plans-sur-Bex 1977), Lecture Notes in Mathematics 685 (Springer-Verlag, 1978).

[7] S. Kojima. A classification of some even dimensional fibred knots. J. Fac. Science Univ. of Tokyo Sec 1A 24 (1977), 671-683.

[8] J. LeVINE. Unknotting theorems in codimension 2. Topology 4 (1965), 9-16.

[9] J. Levine. An algebraic classification of some knots of codimension two. Comment. Math. Helv. 45 (1970), 185-198.

[10] D. LrNes. On odd dimensional fibred knots obtained by plumbing and twisting. London Math. Soc. (in the Press).

[11] H. Morton. Fibred knots with a given Alexander polynomial. Noeuds, Tresses et Singularités (Séminaire des Plans-sur-Bex 1982), Monographie 31, l'Enseignement Mathématique. Genève (1983), 204-222.

[12] S. Smale. On the structure of manifolds. Amer. J. Math. 84 (1962), 387-399.

[13] C. T. C. WALL. Classification problems in differential topology I. Topology 2 (1963), 253-261.

[14] G. Whitehead. Elements of Homotopy Theory. Graduate Texts in Mathematics 61 (Springer. Verlag, 1978). 